\title{
Quality, not just quantity: the role of qualitative methods in anesthesia research
}

\author{
Duminda N. Wijeysundera MD FRCPC, ${ }^{\star} \dagger$ Brian M. Feldman MD MSC FRCPC $† \ddagger \mathbb{S}$
}

I $\mathrm{N}$ this issue of the Journal, Ansermino et al. ${ }^{1}$ present their study describing how anesthesiologists use physiological monitors to identify three critical ventilatory events. To understand clinicians' cognitive processes for identifying these events, the authors began their study by conducting several structured interviews. Subsequently, insights from these interviews guided the development of consensus-based diagnostic criteria.

Readers will note that this paper differs from the randomized controlled trials, cohort studies, case-control studies, cross-sectional studies, or meta-analyses typically seen in the clinical anesthesia literature. There is no mention of specific pre-defined hypotheses, sample size calculations, statistical tests, $P$-values, or 95\% confidence intervals. Instead, the authors used a qualitative approach to answer their research question. What is qualitative research? For readers unfamiliar with this methodology, it might best be described in comparison to the more traditional quantitative research methodology.

Quantitative research generally involves pre-specified hypotheses, collection of quantifiable data (e.g., hospital length of stay, visual analogue pain scores), and statistical analysis of these data to test the hypotheses. Quantitative methods generally perform well when determining whether an intervention has benefit, or when establishing how much a factor influences an outcome. ${ }^{2}$ The importance of quantitative methods is evident by the many important questions in anesthesia practice they have addressed, such as, whether nitrous oxide increases postoperative complications ${ }^{3}$ or how much preoperative risk factors increase the risk of major cardiac events. ${ }^{4}$

However, quantitative methods may be less suited for questions involving the interpretation of human behaviour and social interactions. ${ }^{5}$ Qualitative research questions generally ask what, how, or why, ${ }^{2}$ and these questions have direct relevance for anesthesiology, especially since the perioperative environment is a dynamic multidisciplinary social environment. Pertinent examples of such research questions would include the following: What are the attitudes of healthcare professionals with regard to reporting medication errors? ${ }^{6}$ How do clinicians identify critical ventilatory events? ${ }^{1}$ Why do some patients refuse epidural analgesia? It is questions such as these that might best be answered, at least in part, by using qualitative research methods. ${ }^{5}$

Qualitative research, which has its origin in the social sciences, involves the systematic observation and analysis of human behaviour through methods such as focus groups, in-depth interviews, or passive observation. ${ }^{5}$ Importantly, these methods capture data describing speech or behaviour, in contrast to the numeric data collected in a quantitative study. These collected data are transcribed into text and, subsequently, closely reviewed (using what is termed as 'content analysis') to identify patterns and themes that may explain the observed behaviour or interaction. ${ }^{5}$ Therefore, qualitative research is important for developing new hypotheses (i.e., hypothesis generating), as opposed to testing pre-specified hypotheses (i.e., hypothesis testing). For example, Ansermino et al. conducted in-depth inter-

CAN J ANESTH $2008 / 55: 10 /$ pp 670-673

From the Department of Anesthesia, ${ }^{*}$ Toronto General Hospital, University of Toronto; the Department of Health Policy Management and Evaluation, $\dagger$ University of Toronto; the Department of Public Health Sciences, $\ddagger$ University of Toronto; the Division of Rheumatology, $\$$ and the Department of Pediatrics, $\mathbb{S}$ the Hospital for Sick Children, University of Toronto; Toronto, Ontario, Canada.

Address correspondence to: Dr. Duminda N. Wijeysundera, Department of Anesthesia, Toronto General Hospital \& University of Toronto, EN 3-450, 200 Elizabeth Street, Toronto, Ontario M5G 2C4, Canada. Phone: 416-340-4800, ext. 8981; Fax: 416-340-3698; E-mail: duminda.wijeysundera@uhn.on.ca

Financial support: Dr. Wijeysundera is supported by a Clinician-Scientist Award from the Canadian Institutes of Health Research, and Dr. Feldman is supported by a Canada Research Chair in Childhood Arthritis.

Conflict of interest: No authors have potential conflicts of interest pertaining to this paper. 
views with five expert anesthesiologists about methods used to identify critical ventilatory events. These interviews were transcribed and, subsequently, were closely reviewed (with the aid of computer software) to identify common themes. These themes were then used to construct a new hypothetical decision-making framework for identifying critical events.

There is an increasing recognition of the value of qualitative methods in healthcare research. ${ }^{7-9}$ Qualitative research methods have similar relevance to anesthesiology. They can be used in isolation to answer questions, such as the following, not readily addressed by quantitative methods: What constitutes the conceptual framework anesthesiologists use to identify critical events? They can also be used to complement traditional quantitative approaches. First, qualitative methods may be used to develop hypotheses that are subsequently tested by a quantitative study. For example, focus groups might suggest that children had better anesthetic experiences if parents were more informed about their children's anesthesia. This hypothesis could then be tested in a randomized trial of a new preoperative educational tool for parents. Second, qualitative methods might also be used to inform the design of a quantitative study. For example, prior to initiating a randomized trial of peripheral nerve blockade for improving quality of recovery after total knee replacement, researchers might first conduct focus groups with surgical patients to determine what actually constitutes patient satisfaction with postoperative recovery. Finally, qualitative methods may be used to further explore the results of a quantitative study. For example, researchers could conduct interviews to further explore the reasons why anesthesiologists were unwilling to participate in a randomized trial of epidural analgesia in major surgery. ${ }^{10}$

Qualitative research methods are not without controversy. ${ }^{11}$ Since qualitative research is inherently subjective, physicians, who have traditionally valued numerical data, have viewed it with some suspicion. ${ }^{12}$ Consequently, qualitative researchers have faced challenges when publishing their work in biomedical journals. ${ }^{12}$ The assessment of methodological quality in qualitative research is not straightforward, although recent guidelines may make it easier for readers, reviewers, and journal editors to identify high-quality qualitative research. ${ }^{2,13}$ In addition, the findings of qualitative methods apply specifically to the participants and the setting of the study; therefore, readers must carefully consider whether the conclusions ascertained in the research setting can necessarily be extrapolated in relation to other populations. ${ }^{14}$

Despite these issues, it is clear that qualitative research is a valuable addition to traditional methodologies in anesthesia research. Some challenges for the specialty include: taking a qualitative approach into account when addressing specific research questions; involving expert qualitative researchers in anesthesia research teams; and training clinician-scientists in qualitative methodology. The depth and breadth of the anesthesia literature will certainly be improved by meeting these challenges. After all, research should comprise both quantity and quality.

\section{Non seulement la quantité, mais aussi la qualité : le rôle des méthodes qualitatives dans la recherche en anesthésie}

Dans ce numéro du Journal, Ansermino et coll. ${ }^{1}$ présentent leur étude qui décrit comment les anesthésiologistes utilisent les moniteurs physiologiques pour identifier trois événements respiratoires critiques. Afin de comprendre les processus cognitifs auxquels les cliniciens font appel pour identifier ces événements, les auteurs ont débuté leur étude en menant plusieurs entretiens structurés. Par la suite, les perspectives tirées de ces entretiens ont guidé l'élaboration de critères de diagnostic basés sur le consensus.

Le lecteur remarquera que cet article diffère des études randomisées contrôlées, de cohorte, castémoins ou transversales, mais aussi des méta-analyses qui sont généralement l'apanage de la littérature anesthésique clinique. Aucune mention n'est faite d'hypothèses spécifiques prédéfinies, de calcul de la taille de l'échantillon, de tests statistiques, de valeurs $P$, ou d'intervalles de confiance de $95 \%$. Au lieu de cela, les auteurs ont privilégié une approche qualitative pour répondre à leur question de recherche. Qu'estce que la recherche qualitative ? Pour le lecteur peu familier avec ce type de méthodologie, cette approche est peut-être décrite de façon optimale lorsqu'on la compare à la méthodologie de recherche quantitative, plus conventionnelle. 
Une recherche quantitative implique généralement des hypothèses spécifiées au préalable ainsi que la collecte de données quantifiables (par ex., la durée de séjour à l'hôpital, des échelles visuelles analogiques de douleur), et une analyse statistique de ces données pour tester la validité des hypothèses. En général, les méthodes quantitatives donnent de bons résultats lorsqu'on tente de déterminer si une procédure a des avantages, ou lorsqu'on tente d'établir dans quelle mesure un facteur influence un devenir. ${ }^{2}$ L'importance des méthodes de recherche quantitatives est évidente, comme le prouvent les nombreuses questions fondamentales qu'elles ont abordées dans la pratique anesthésique. Par exemple, elles ont permis de déterminer si l'oxyde nitrique augmentait les complications postopératoires ${ }^{3}$ ou dans quelle mesure les facteurs de risque préopératoires augmentaient le risque de complication cardiaque grave. ${ }^{4}$

Cependant, il se peut que les méthodes quantitatives soient moins efficaces dans le cas de questions touchant à l'interprétation des comportements humains ou aux interactions sociales. ${ }^{5}$ En général, la recherche qualitative cherche à savoir quoi, comment, ou pourquo $i^{2}$ et ces questions sont particulièrement pertinentes en anesthésiologie, étant donné que l'environnement périopératoire est un environnement social à la fois dynamique et pluridisciplinaire. Voici quelques exemples pertinents de telles questions de recherche: Quelles sont les attitudes des professionnels de la santé quant au signalement des erreurs de médication $?^{6}$ Comment les cliniciens identifient-ils les événements respiratoires critiques ?' ${ }^{1}$ Pourquoi certains patients refusent-ils l'analgésie péridurale ? Les méthodes de recherche qualitative permettent de mieux répondre à ce type de questions, du moins en partie. $^{5}$

La recherche qualitative, qui prend son origine dans les sciences sociales, implique l'observation et l'analyse systématiques des comportements humains par le biais de méthodes tels que les groupes de discussion, les entretiens approfondis, ou l'observation passive. ${ }^{5} \mathrm{Il}$ est important de noter que ces méthodes compilent des données décrivant la parole ou le comportement, contrairement aux données numériques collectées dans une étude quantitative. Les données collectées sont retranscrites par écrit, puis analysées en profondeur (à l'aide de ce qu'on appelle une " analyse de contenu »), afin d'identifier des tendances et des thèmes qui pourraient expliquer le comportement ou l'interaction observés. ${ }^{5}$ Pour cette raison, la recherche qualitative joue un rôle prépondérant dans l'élaboration de nouvelles hypothèses (c.-à-d. la génération d'hypothèses), plutôt que dans le test d'hypothèses spécifiées au préalable (c.-à-d. le test d'hypothèses). Par exemple, Ansermino et coll. ont mené des entretiens approfondis auprès de cinq anesthésiologistes experts concernant les méthodes utilisées pour identifier les événements respiratoires critiques. Ces entretiens ont été retranscrits puis analysés avec soin (à l'aide de logiciels informatiques) pour identifier les thèmes communs. Ces thèmes ont ensuite été utilisés pour élaborer un nouveau cadre de prise de décision hypothétique pour l'identification des événements critiques.

La valeur des méthodes qualitatives dans la recherche en soins de santé est de plus en plus reconnue. ${ }^{7-9}$ Les méthodes de recherche qualitative sont tout aussi pertinentes en anesthésiologie. Elles peuvent être utilisées de façon isolée pour répondre à des questions qu'il est difficile d'aborder avec des approches quantitatives, comme par exemple : Qu'est-ce qui constitue le cadre conceptuel utilisé par les anesthésiologistes pour identifier les événements critiques ? Ces méthodes peuvent également être utilisées pour compléter les approches quantitatives conventionnelles. En premier lieu, on peut avoir recours à des méthodes qualitatives pour élaborer des hypothèses qui seront par la suite testées dans le cadre d'une étude quantitative. Par exemple, des groupes de discussion pourraient suggérer que l'expérience anesthésique de patients pédiatriques serait améliorée si les parents étaient mieux informés au sujet de l'anesthésie de leur enfant. Cette hypothèse pourrait ensuite être testée dans une étude randomisée portant sur un nouvel outil éducatif préopératoire destiné aux parents. Deuxièmement, les méthodes qualitatives pourraient également être utilisées pour orienter la conception d'une étude quantitative. Par exemple, avant d'initier une étude randomisée portant sur le recours à un bloc nerveux périphérique dans le but d'améliorer la qualité de la récupération après une arthroplastie totale du genou, les chercheurs pourraient commencer par mener des discussions de groupe avec des patients chirurgicaux afin de déterminer exactement quels critères sont considérés importants par les patients pour qu'ils se considèrent satisfaits de leur récupération postopératoire. Enfin, les méthodes qualitatives pourraient également être utilisées pour explorer plus en détail les résultats d'une étude quantitative. Par exemple, les chercheurs pourraient mener des entretiens pour explorer plus en détail les raisons pour lesquelles certains anesthésiologistes ne désirent pas participer à une étude randomisée évaluant l'analgésie péridurale dans les chirurgies majeures. ${ }^{10}$

Les méthodes de recherche qualitative demeurent toutefois controversées. ${ }^{11}$ Étant donné que la recherche qualitative est subjective en soi, les médecins, qui 
attachent traditionnellement une grande valeur aux données numériques, la considèrent d'un oeil soupçonneux. ${ }^{12}$ Pour cette raison, les chercheurs qualitatifs font face à un certain nombre de défis lorsqu'ils tentent de publier leurs travaux dans les revues biomédicales. ${ }^{12}$ L'évaluation de la qualité méthodologique dans les études qualitatives n'est pas évidente, bien que certaines directives récentes pourraient faciliter l'identification d'une recherche qualitative de bonne qualité pour le lecteur, le réviseur, et le rédacteur de la revue. ${ }^{2,13}$ De plus, les résultats issus de recherches qualitatives s'appliquent spécifiquement aux participants et au contexte de l'étude ; le lecteur doit en être conscient, et juger par lui-même si les conclusions tirées du contexte de la recherche peuvent être ou non extrapolées et appliquées à d'autres populations. ${ }^{14}$

En dépit de toutes ces questions, il est clair que la recherche qualitative constitue un ajout inestimable aux méthodologies conventionnelles utilisées en recherche anesthésique. Parmi les défis auxquels notre spécialité sera confrontée, citons : la prise en compte d'une approche qualitative lorsqu'on aborde certaines questions de recherche spécifiques; la participation de chercheurs qualitatifs experts dans les équipes de recherche anesthésique ; et la formation des scientifiques cliniciens en méthodologie qualitative. Si nous parvenons à relever ces défis, l'envergure et la qualité de la littérature anesthésique ne peuvent qu'en être améliorées. Après tout, la recherche devrait inclure à la fois quantité et qualité.

\section{References}

1 Ansermino JM, Dosani M, Amari E, Choi PT, Schwarz $S K$. Defining rules for the identification of critical ventilatory events. Can J Anesth 2008; 55: 702-14.

2 Giacomini MK, Cook DJ. Users' guides to the medical literature: XXIII. Qualitative research in health care A. Are the results of the study valid? Evidence-Based Medicine Working Group. JAMA 2000; 284: 357-62.

3 Myles PS, Leslie K, Chan MT, et al. Avoidance of nitrous oxide for patients undergoing major surgery: a randomized controlled trial. Anesthesiology 2007; 107: 221-31.

4 Lee TH, Marcantonio ER, Mangione CM, et al. Derivation and prospective validation of a simple index for prediction of cardiac risk of major noncardiac surgery. Circulation 1999; 100: 1043-9.

5 Pope C, Mays N. Reaching the parts other methods cannot reach: an introduction to qualitative methods in health and health services research. BMJ 1995; 311 : 42-5.

6 Sanghera IS, Franklin BD, Dhillon S. The attitudes and beliefs of healthcare professionals on the causes and reporting of medication errors in a UK intensive care unit. Anaesthesia 2007; 62: 53-61.

7 Berkwits M, Aronowitz R. Different questions beg different methods. J Gen Intern Med 1995; 10: 409-10.

8 Inmi TS. The virtue of qualitative and quantitative research. Ann Intern Med 1996; 125: 770-1.

9 Jones $R$. Why do qualitative research? BMJ 1995; 311 : 2.

10 Beattie WS, Choi PT, Yang HY, Buckley N, Bryson G, Paul G. Results of the perioperative epidural trial pilot study. Can J Anesth 2008; 55: 475494 (abstract).

11 Poses RM, Isen AM. Qualitative research in medicine and health care: questions and controversy. J Gen Intern Med 1998; 13: 32-8.

12 Greenhalgh T, Taylor $R$. Papers that go beyond numbers (qualitative research). BMJ 1997; 315: 740-3.

13 Giacomini MK, Cook DJ. Users' guides to the medical literature: XXIII. Qualitative research in health care B. What are the results and how do they help me care for my patients? Evidence-Based Medicine Working Group. JAMA 2000; 284: 478-82.

14 Sinuff T, Cook DJ, Giacomini M. How qualitative research can contribute to research in the intensive care unit. J Crit Care 2007; 22: 104-11. 